\title{
Avenanthramide-C reduces the viability of MDA-MB-231 breast cancer cells through an apoptotic mechanism
}

\author{
Jordan Hastings and Jason Kenealey* ${ }^{*}$
}

\begin{abstract}
Background: Avenanthramides (AVN) are a relatively unstudied family of phytochemicals that could be novel chemotherapeutics. These compounds, found in oats, are non-toxic to healthy cells and have been shown to reduce viability of human colon and liver cancers in vitro. However, these studies do not elucidate a molecular mechanism for individual AVN. In this study we aim to see the effects of AVN on MDA-MB-231 breast cancer cells.

Methods: An MTT assay was used to determine cell viability. Staining and analysis with a flow cytometer was used to identify cell cycle progression and apoptosis. Flo Jo software was used to analyze the cytometric data. In all experiments, statistical significance was determined by a two-tailed t test.

Results: This study demonstrates that AVN-A, B, and C individually reduce viability in the MDA-MB-231 breast cancer cell line. AVN-C has the most potent decrease in tumor cell viability, decreasing viable cells to below $25 \%$ at $400 \mu \mathrm{M}$ when compared to control after $96 \mathrm{~h}$. We demonstrate that treatment with AVN-C causes DNA fragmentation and accumulation of over $90 \%$ of cells into a sub $\mathrm{G}_{1}$ cell cycle population. Further, we conclude that AVN-C treated cells activate apoptosis because $97 \%$ of treated cells stain positive for annexin $\vee$ while $91 \%$ have caspase-3/7 activity, a late marker of apoptosis.
\end{abstract}

Conclusions: Breast cancer cells treated with AVN-C have a decrease in cell viability, an increase in the sub $\mathrm{G}_{1}$ population, and stain positive for both annexin $\mathrm{V}$ and caspase activity, indicating that AVN-C induces apoptosis in breast cancer cells. These compounds may be able to act as chemotherapeutics as demonstrated through future in vivo studies.

Keywords: Avenanthramides, Oats, Breast cancer, Apoptosis

\section{Background}

In the US, nearly one in eight women will develop invasive breast cancer over the course of their life. In 2017, breast cancer is expected to account for nearly $30 \%$ of newly diagnosed cancers in woman, and result in over 40,000 deaths. For US women, breast cancer has a higher death rate than any other cancer, besides lung cancer [1]. Over the past two decades, scientists have determined the mechanistic characteristics of cancer as outlined in the 'Hallmarks of Cancer' described by Hanahan

\footnotetext{
*Correspondence: Jason_kenealey@byu.edu

Department of Nutrition, Dietetics, and Food Science, Brigham Young University, ESC S249, Provo, UT 84602, USA
}

and Weinberg in 2000 and later added to in 2011; some of these hallmarks include self-sufficiency in growth signaling, insensitivity to anti-growth signals, evading apoptosis, genome instability and mutation, and tumor promoting inflammation [2, 3].

Avenanthramides (AVN) are a family of phytochemicals found in oats. There are three main isoforms of the chemical simply classified as avenanthramide A, B, and C (Fig. 1) [4]. Currently available literature demonstrates that AVN have chemotherapeutic potential due to its effects on some of the hallmarks of cancer. First, AVN have anti-inflammatory properties [5] and by modulating pro-inflammatory cytokines AVN could modulate the tumor microenvironment and decrease tumor size. 


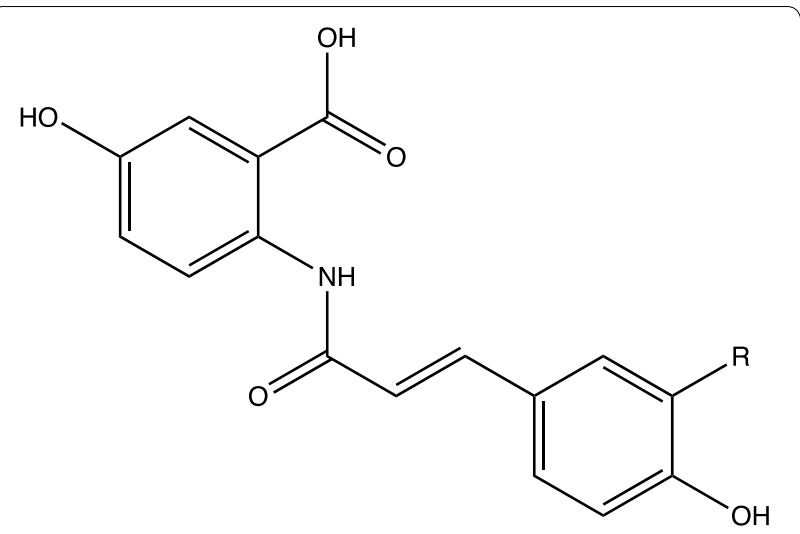

\begin{tabular}{ll} 
Compound & $\mathrm{R}$ \\
\hline AVN-A & $\mathrm{H}$ \\
AVN-B & $\mathrm{OCH} 3$ \\
AVN-C & $\mathrm{OH}$
\end{tabular}

Fig. 1 The structure of $A V N-A, B$ and $C$

Additionally, AVN can affect cell cycle progression and have been shown to arrest cells by decreasing cyclins $[6$, 7]. The ability to affect cyclin levels, along with documented decreases in viability have led to the understanding that AVN-C is anti-proliferative [8, 9]. Alternatively, a mixture of all three AVN isoforms activated pro-apoptotic mechanisms such as caspases, but the ability of each AVN isoform to induce apoptosis has not been evaluated [10].

Current cancer treatments, though moderately effective, attack both cancerous and non-cancerous cells, resulting in dose-limiting side effects. Conversely, diets consisting of AVN enriched oat extract have shown no toxicity in both animal and human models [11]. In addition, unlike many other plant-based molecules, AVN have high bioavailability, making them potential chemotherapeutics. In human trials the maximum plasma concentration $\left(\mathrm{C}_{\max }\right)$ of $\mathrm{AVN}-\mathrm{A}, \mathrm{B}$, and $\mathrm{C}$ have been reported at a range of $166.7-1002.2 \mathrm{nmol} / \mathrm{L}$ for AVN-A, 49.3$153.5 \mathrm{nmol} / \mathrm{L}$ for $\mathrm{AVN}-\mathrm{B}$, and $29.6-328.1 \mathrm{nmol} / \mathrm{L}$ for AVN-C when participants consumed $1 \mathrm{~g}$ of avenanthramide enriched oat extract which contained 154, 109, and $117 \mu \mathrm{M} / \mathrm{g}$ of AVN-A, B and C respectively [12]. Notably, when ingesting half the amount of AVN $C_{\max }$ fell more than half, suggesting that higher levels of consumption may lead to even higher $C_{\max }$ levels.

In this study, we tested the effects of AVN A, B, and $C$ on MDA-MB-231 breast cancer cells and found that all three AVN decrease breast cancer cell viability in a time and concentration dependent manner; $\mathrm{AVN}-\mathrm{C}$ is the most potent of the three compounds. Furthermore, we demonstrate that the decrease in viability induced by $\mathrm{AVN}-\mathrm{C}$ occurs through an apoptotic mechanism as measured through multiple methods of flow cytometric analysis.

\section{Materials and methods}

Avenanthramides $\mathrm{A}, \mathrm{B}$, and $\mathrm{C}$ were purchased from Sigma Aldrich (St. Louis, MO) and stocks were diluted to $60 \mathrm{mM}$ by DMSO. MDA-MB-231 human breast cancer (HTB-26) cell line was purchased from ATCC (Manassas, VA). The passage numbers of cells used in experiments ranged from 5 to 25 . MTT (3-(4,5-dimethylthiazol-2-yl)2,5-diphenyltetrazolium bromide) was purchased from Acros Organics (Morris Plains, NJ). MTT solvent was made up in the lab using $4 \mathrm{mM} \mathrm{HCl}, 0.1 \%$ Nonidet P-4 (NP40) in isoproponol. Propidium iodide (PI) was purchased from Cayman Chemical (Ann Arbor, MI). FITC Annexin V Apoptosis Detection Kit (556547) was purchased from BD Biosciences (San Jose, CA). CellEvent Caspase 3/7 Green Flow Cytometry Assay Kit was purchased from Thermo Fisher Scientific (Waltham, MA) (C10427).

\section{Cell culture}

MDA-MB-231 cells were cultured in Dulbecco's Modified Eagle Medium (DMEM). DMEM was supplemented with $10 \%$ heat-inactivated fetal bovine serum (FBS) and $1 \%$ penicillin/streptomycin. The cell line was cultured at $37{ }^{\circ} \mathrm{C}$ in $5 \% \mathrm{CO}_{2}$. Cells were subcultured by treatment with trypsin upon reaching near confluency and reseeded by placing one million cells into a fresh T-75 culture plate.

\section{Cell viability analysis}

Cell viability was determined using an MTT assay. Cell were plated at 10,000 cells per well in a 96-well plate. Cells were incubated for $24 \mathrm{~h}$ at $37^{\circ} \mathrm{C}$ to allow for adhesion, at which time the DMEM was removed and new media with individual avenanthramide analogues at the indicated concentration was added. After the indicated treatment period was reached $(48-96 \mathrm{~h}) 20 \mu \mathrm{L}$ of $5 \mathrm{mg} /$ $\mathrm{mL}$ MTT was added to each well. Cells were again incubated for $3.5 \mathrm{~h}$ at $37{ }^{\circ} \mathrm{C}$ in a $5 \% \mathrm{CO}_{2}$ culture hood. The media and MTT was removed and $150 \mu \mathrm{L}$ of MTT solvent was added. Cells were agitated on an orbital shaker at $75 \mathrm{rpm}$ for $15 \mathrm{~min}$ before absorbance was read at $590 \mathrm{~nm}$ with a reference filter at $620 \mathrm{~nm}$.

\section{Cell cycle analysis}

Cells were plated at 100,000 cells per well in a 12-well plate and allowed to adhere for $24 \mathrm{~h}$. DMEM was removed and new culture media with the indicated concentrations of each avenanthramide was added. After a 96-h treatment period cells were collected and pelleted 
down. Pellets were washed once with $1 \times$ PBS. $500 \mu \mathrm{L}$ of $70 \%$ ethanol was added dropwise while vortexing and cells were placed in $4{ }^{\circ} \mathrm{C}$ overnight. The cells were then pelleted down and the ethanol was removed. The pellet was washed with $1 \times$ PBS before adding $200 \mu \mathrm{L}$ PI made up at $50 \mu \mathrm{g} / \mathrm{mL}$ in $1 \times$ PBS. Samples were analyzed with a BD Accuri Flow Cytometer for 50,000 counts.

\section{Annexin $\mathrm{V}$ and $\mathrm{PI}$ apoptosis analysis}

Cells were plated at 100,000 cells per well in a 12-well plate and allowed to adhere for $24 \mathrm{~h}$. DMEM was removed and new culture media with the indicated concentrations of each avenanthramide was added. After a 96-h treatment period cells were harvested and subjected to centrifugation. Staining was performed per the manufacturer's instructions (BD Biosciences). Briefly, cells were pelleted down and washed twice with cold PBS before being resuspended in $1 \times$ binding buffer at $1 \times 10^{6}$ cells/mL. $5 \mu \mathrm{L}$ FITC annexin V and PI were added to each sample. Samples were gently vortexed then incubated for 15 min in the dark before $400 \mu \mathrm{L} 1 \times$ binding buffer was added to each tube. Samples were analyzed with a BD Accuri Flow Cytometer for 50,000 counts.

\section{CellEvent caspase 3/7 green flow cytometry apoptosis analysis}

Cells were plated at 50,000 cells per well in a 12-well plate and allowed to adhere for $24 \mathrm{~h}$. DMEM was removed and new culture media with indicated concentrations of each avenanthramide was added. After $96 \mathrm{~h}$ treatment period media and cells were harvested and centrifuged down. Fluorescent labels were added per the manufacturer's instructions (Thermo Fisher). Briefly, $1 \mu \mathrm{L}$ of CellEvent Caspase-3/7 Green Detection Reagent was added to all samples which were then incubated for $30 \mathrm{~min}$. During the last $5 \mathrm{~min}$ of incubation $1 \mu \mathrm{L}$ of the SYTOX AADvanced dead cell stain solution was added to each sample. Samples were analyzed with a BD Accuri Flow Cytometer for 50,000 counts.

\section{Analysis of flow cytometric data}

Data obtained from the flow cytometer was analyzed with FlowJo software using unstained cells and cell samples stained with one marker only to set fluorescence compensations for each assay.

\section{Statistical significance}

In all experiments, statistical significance was determined by a two-tailed $t$ test. Significant differences in cell viability between DMSO negative control vehicle and treatment (either AVN or staurosporine positive control for activating apoptosis) are indicated by * if $\mathrm{p}<0.05$, ** if $\mathrm{p}<0.01$ and $* * *$ if $\mathrm{p}<0.001$. For cell viability experiments significance was determined between vehicle and each AVN concentration after normalization. For cell cycle analysis experiments significance was determined by comparing each stage of the cell cycle after AVN treatment with the same stage from each vehicle control treatment. For all quadrant based flow cytometry experiments significance was determined by normalizing the quadrants and then comparing each vehicle quadrant to its treated counterpart.

\section{Results}

Avenanthramides decrease breast cancer viability in vitro To determine if AVN-A, B, and C have chemotherapeutic potential we first utilized an MTT cell viability assay. The effect of AVN-A, AVN-B and AVN-C on breast cancer cell viability is shown in Figs. 2, 3 and 4, respectively. Viability was assessed at, 50, 100, 200, and $400 \mu \mathrm{M}$ treatments for AVN-A, B, and C at 48, 72, and $96 \mathrm{~h}$ timepoints. AVN-A was the first to show significance at the $400 \mu \mathrm{M}$ concentration after $48 \mathrm{~h}$ (Fig. 2a). AVN-B and $\mathrm{C}$ didn't show significance at the same concentration until the 96-h timepoint (Figs. 3c, 4c). Significant differences were noted in all three AVN treatments after $96 \mathrm{~h}$ with AVN-A remaining significant only at the highest dose, while AVN-B showed significance at both 200, and $400 \mu \mathrm{M}$ and AVN-C showed significance as low as $50 \mu \mathrm{M}$ after $96 \mathrm{~h}$. Although AVN-C showed significance at a later time point than $\mathrm{A}$ or $\mathrm{B}$, its observed decrease in viability was twice that of its counterparts. It is clear that AVN-C has a dramatic effect on breast cancer cell viability, while AVN-A and B display modest results, albeit sooner. Because of the observed differences in both timing and degree of decreased viability from the different treatments we hypothesized that different mechanisms may be at play and expected to see varying results from the treatments in further testing. Due to the fact that significance was observed in all three treatments only at $400 \mu \mathrm{M}$ after $96 \mathrm{~h}$, all following studies were conducted at this time and concentration unless noted otherwise.

\section{Avenanthramide-C causes DNA fragmentation}

We hypothesized that AVN may be decreasing cell viability by either inhibiting the cell cycle or activating apoptosis in the tumorigenic cells. As a result, we determined the percentage of cells in each stage of the cell cycle after $96 \mathrm{~h}$ of treatment with AVN at $400 \mu \mathrm{M}$ (Fig. 5). PI staining revealed no significant difference between the vehicle and AVN-A and B for any stage of the cell cycle. Vehicle control showed $9.26 \%$ cells sub $G_{1}, 69.53 \%$ cells in the $G_{1}$ stage, $9.23 \%$ cells in the $S$ stage, and $11.97 \%$ cells in the $G_{2}$ stage. AVN-A treatment resulted in $15.79 \%$ cells sub $G_{1}$ $(p=0.42), 62.85 \%$ cells in the $G_{1}$ stage $(p=0.25), 10.96 \%$ cells in the $S$ stage $(p=0.57)$, and $10.4 \%$ cells in the $G_{2}$ 

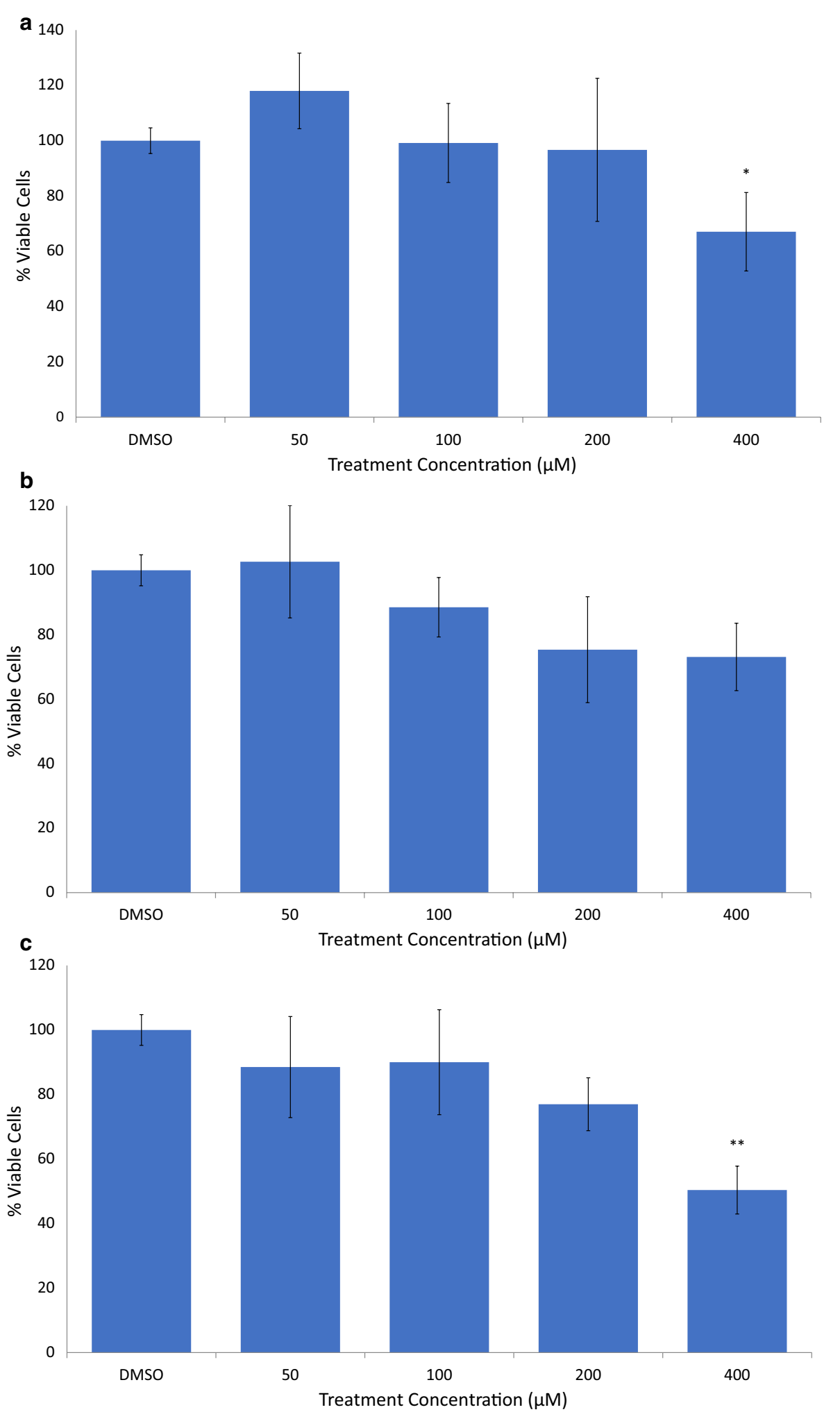

Fig. 2 AVN-A decrease viability of MDA-MB-231 breast cancer cells in a time and concentration dependent manner. a MTT cell viability assay after treatment with DMSO $0.4 \%$ (vehicle) or AVN-A for $48 \mathrm{~h}$ at the indicated concentrations. b AVN-A treatment, $72 \mathrm{~h}$. c AVN-A treatment, $96 \mathrm{~h}$. Averages of a biological replicate done in triplicate; ${ }^{*}$ indicates statistical significance difference from $0.4 \%$ DMSO negative control as determined by a t test at $p<0.05$ level of significance, ${ }^{* *}$ indicates significance level at $p<0.01,{ }^{* * *}$ indicates significance at $p<0.001$ level 

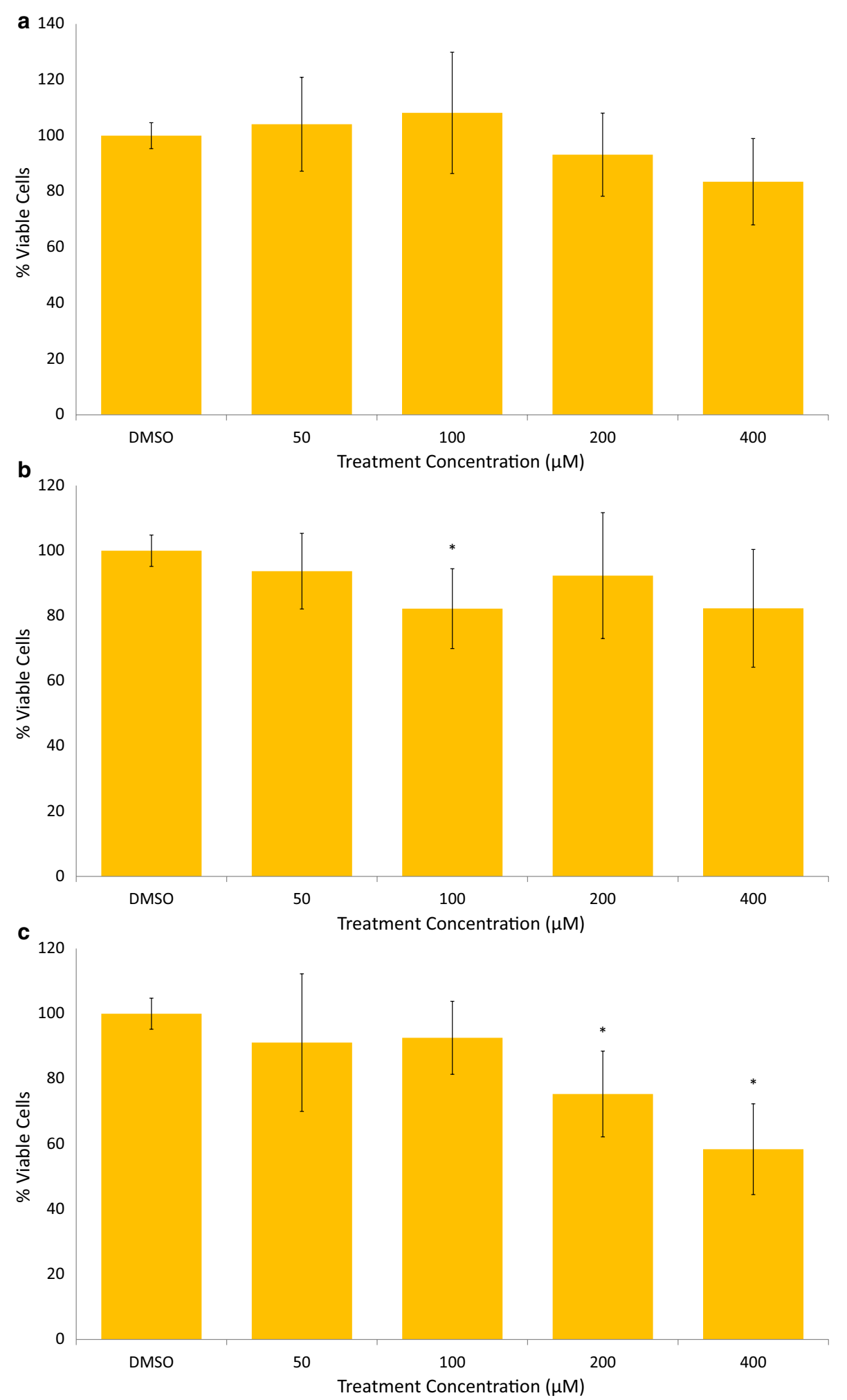

Fig. 3 AVN-B decrease viability of MDA-MB-231 breast cancer cells in a time and concentration dependent manner. a AVN-B treatment, $48 \mathrm{~h}$. b AVN-B treatment, 72 h. c AVN-B treatment, 96 h. Averages of a biological replicate done in triplicate; * indicates statistical significance difference from $0.4 \%$ DMSO negative control as determined by a t test at $p<0.05$ level of significance, ${ }^{* *}$ indicates significance level at $p<0.01,{ }^{* * *}$ indicates significance at $p<0.001$ level 


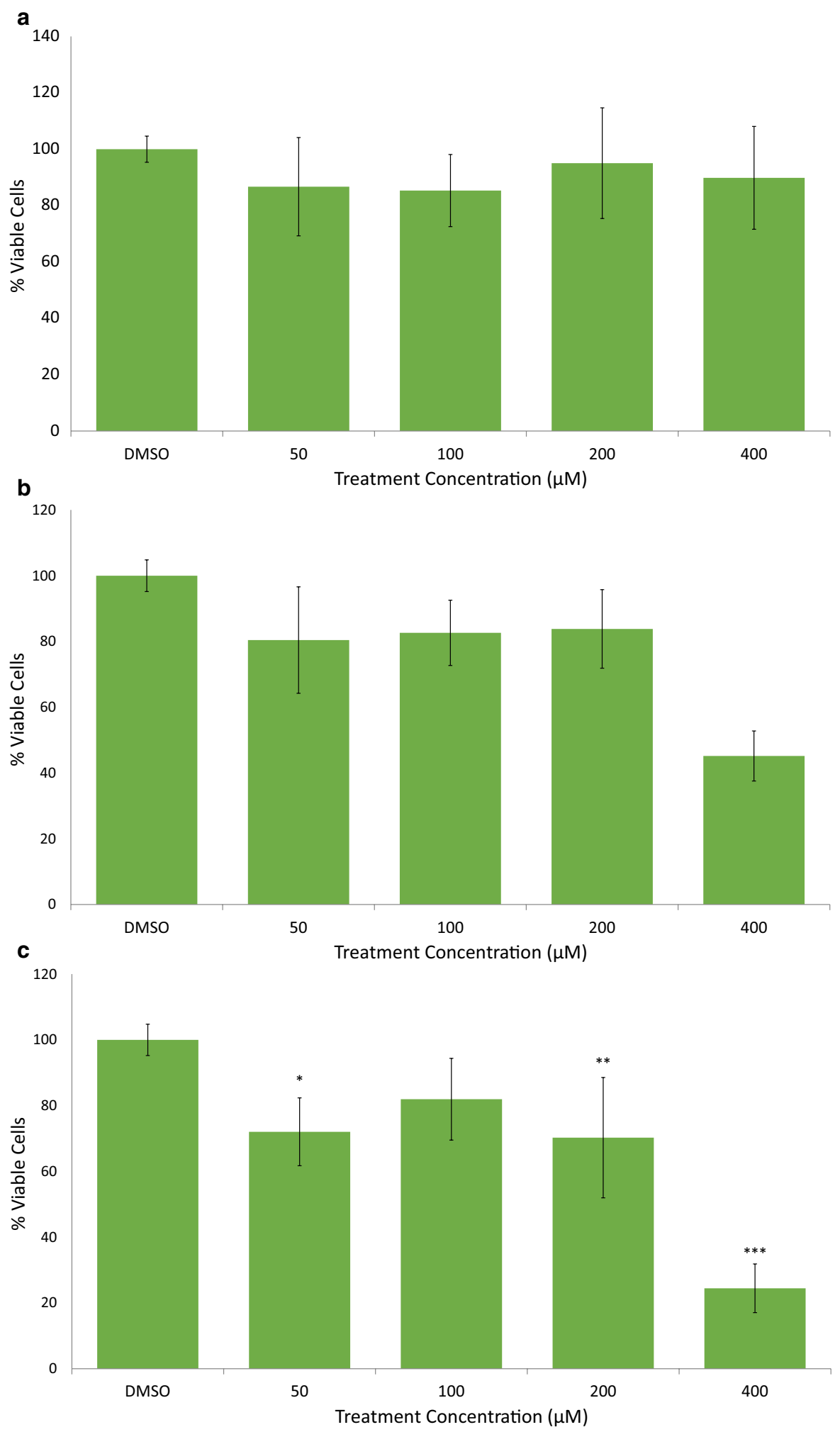

Fig. 4 AVN-C decrease viability of MDA-MB-231 breast cancer cells in a time and concentration dependent manner. a AVN-C treatment, $48 \mathrm{~h}$. b AVN-C treatment, 72 h. c AVN-C treatment, 96 h. Averages of a biological replicate done in triplicate; ${ }^{*}$ indicates statistical significance difference from $0.4 \%$ DMSO negative control as determined by a t test at $p<0.05$ level of significance, ${ }^{* *}$ indicates significance level at $p<0.01,{ }^{* * *}$ indicates significance at $p<0.001$ level 
a
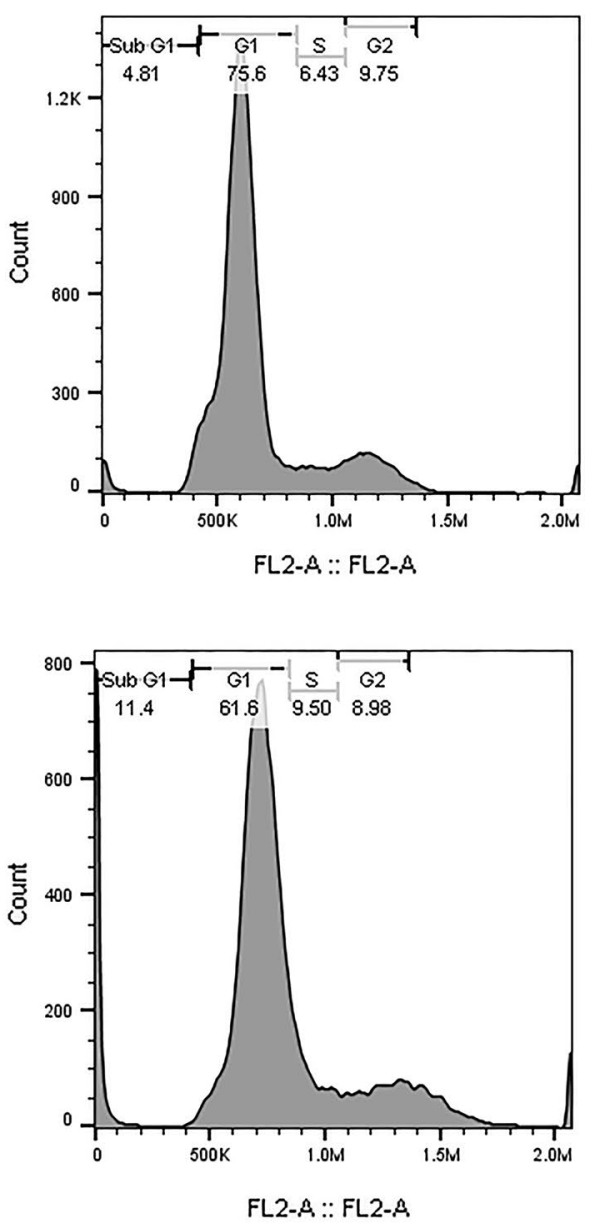

b

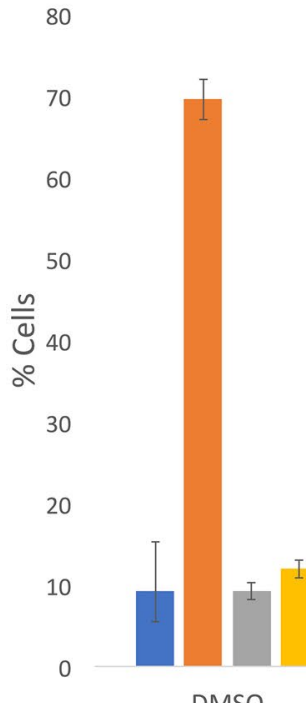

DMSO
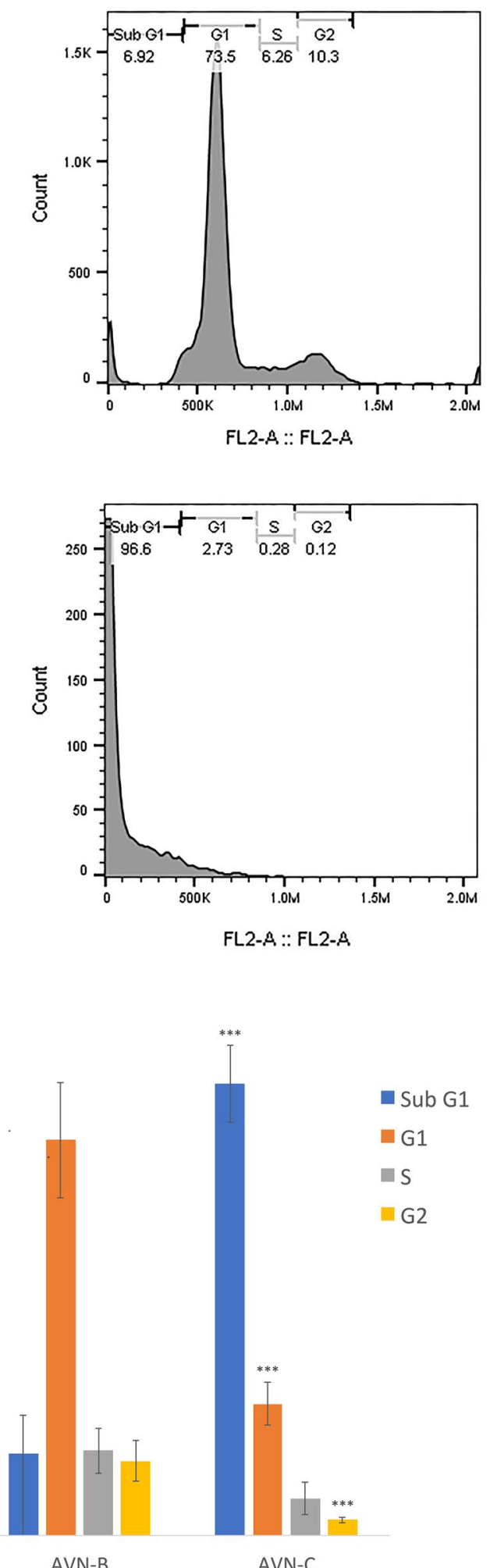

Fig. 5 AVN-C causes DNA fragmentation and an accompanying accumulation of cells in a sub $\mathrm{G}_{1}$ population a Representative sample of flow cytometric analysis of cell cycle after fixation and staining with PI after treatment with DMSO 0.4\% (vehicle) or AVN-A, B, or C at $400 \mu \mathrm{M}$ for $96 \mathrm{~h}$. b Graphical representation of average \% cells in each stage of the cell cycle for three biological replicates done in triplicate. ${ }^{*}$ Indicates statistical significance difference (when comparing each stage of the cell cycle) from $0.4 \%$ DMSO negative control as determined by a t test at $p<0.05$ level of significance, ${ }^{* *}$ indicates significance level at $p<0.01,{ }^{* * *}$ indicates significance at a $p<0.001$ level 


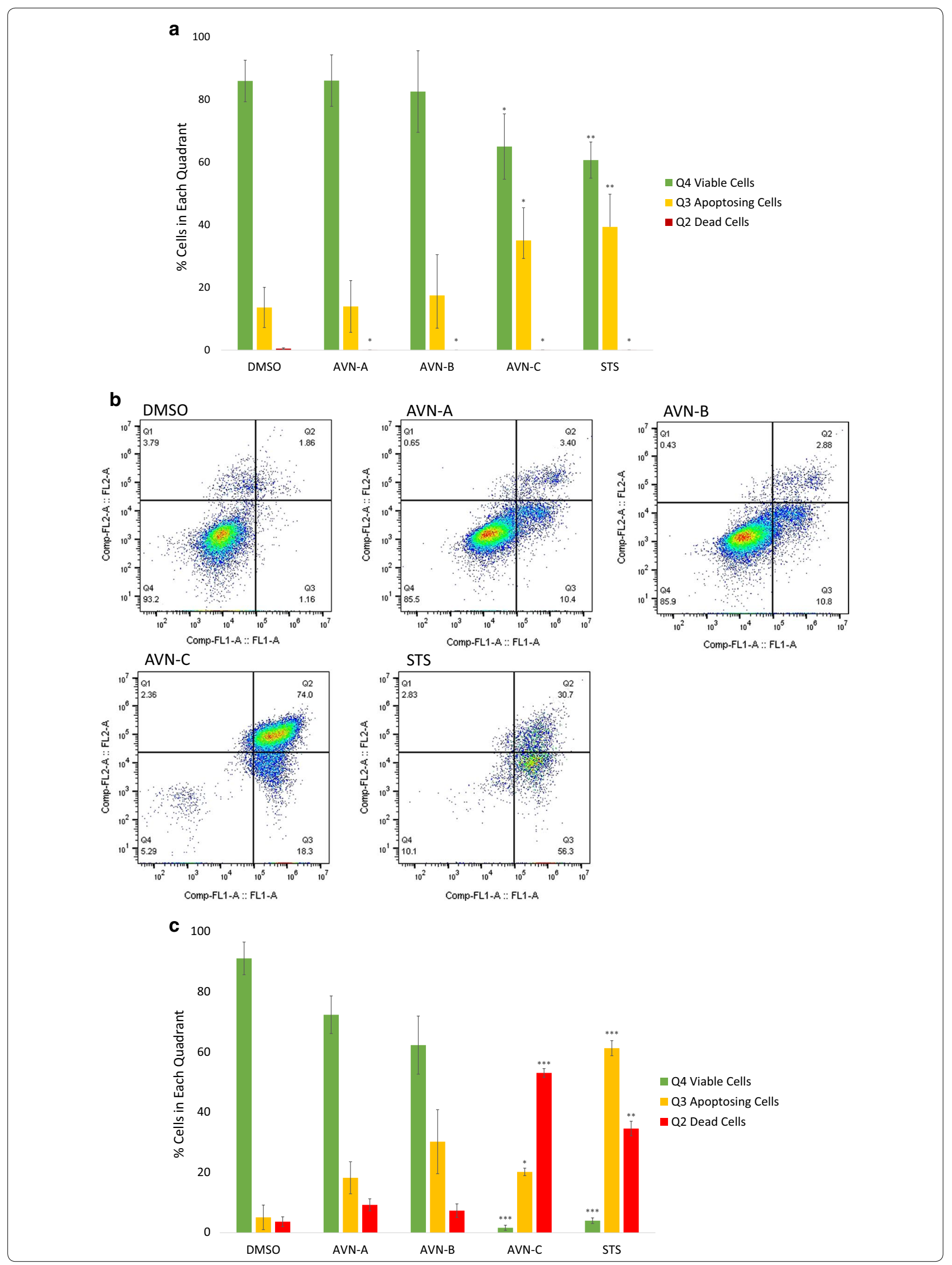


Fig. 6 Treatment with AVN-C causes breast cancer cells to stain positive for annexin V. a Graphical representation of the percentage of cells in each quadrant after staining with $\mathrm{Pl}$ and annexin $\mathrm{V}$ as done in biological replicate for 48-h treatment. b Representative sample of flow cytometric analysis of apoptosis after cells were stained with PI (Comp-Fl2-A) and annexin V (Comp-FL1-A) after treatment with vehicle DMSO 0.4\%, AVN-A, B, C at $400 \mu \mathrm{M}$, or positive control STS for $96 \mathrm{~h}$. c Graphical representation of the percentage of cells in each quadrant as described in (a). Results are a biological replicate done in triplicate for 96 -h treatment. ${ }^{*}$ Indicates statistical significance difference from $0.4 \%$ DMSO negative control for each individual quadrant as determined by a t test at $p<0.05$ level of significance, ${ }^{* *}$ indicates significance level at $p<0.01,{ }^{* * *}$ indicates significance at $p<0.001$ level

stage $(\mathrm{p}=0.48)$. AVN-B treatment caused $14.2 \%$ cells sub $G_{1}(p=0.46), 62.49 \%$ cells in the $G_{1}$ stage $(p=0.14)$, $12.75 \%$ cells in the $S$ stage $(\mathrm{p}=0.19)$, and $10.56 \%$ cells in the $G_{2}$ stage $(p=0.60)$. The cells seemed to be replicating normally despite treatment and a decrease in viability. AVN-C however showed a massive accumulation of cells in a sub $G_{1}$ population $(71.03 \%$ of cells, $p=1.14 \mathrm{E}-5)$ resulting in significant changes to the $G_{1}(20.69 \%$ of cells, $p=2.18 \mathrm{E}-6)$, and $\mathrm{G}_{2}(2.44 \%$ of cells, $\mathrm{p}=2.52 \mathrm{E}-4)$ stages and a moderate but insignificant change to the $\mathrm{S}$ stage $(5.85 \%$ of cells, $\mathrm{p}=0.20)$.

The increase in the sub $G_{1}$ population shows that AVN-C is causing DNA fragmentation; this is indicative of an apoptotic mechanism. It is of note that the AVN-C dependent viability decrease $(75.6 \%)$ is nearly identical to the percent of cells found in the Sub $\mathrm{G}_{1}$ population (71\%). We theorize that AVN-C works by activating apoptosis in this cell line. With no changes in cell cycle distribution for AVN-A and B their mechanism of action remained unclear. We observed an increase in sub $G_{1}$ cells for both AVN-A and B that was accompanied by a decrease of cells in the $G_{1}$ phase of equal magnitude, but these changes were not significant.

\section{Avenanthramide-C activates apoptosis in breast cancer cells}

To confirm that AVN-C was activating apoptosis we conducted two experiments. First, we co-stained treated cells with PI and annexin V and second, we analyzed the caspase activity present in each sample. In both experiments we compared our treatments to a DMSO vehicle dose given at the maximum AVN treatment concentration and a treatment with a $0.75 \mu \mathrm{M}$ staurosporine (STS), a known apoptosis activator.

When staining with annexin V and PI we observed a shift from viable cells found in quadrant (Q) 4 to apoptotic and dead cells quadrants (Q3 and Q2 respectively) (Fig. 6); this occurred in all treatment groups and at both 48 and $96-\mathrm{h}$ time points. After $48 \mathrm{~h}$ of treatment AVN-C and STS showed 35\% ( $\mathrm{p}=0.04)$ and $39 \%(\mathrm{p}=0.0076)$ annexin $\mathrm{V}$ positive $(\mathrm{Q} 3+\mathrm{Q} 4)$ cells respectively, while AVN-A and B had no significant changes in annexin positive cells (Fig. 6a). At the same time, the treatment with the most cells in Q2 was AVN-A at 0.02\%. However, when compared to vehicle $(0.46 \%$ of cells in Q2) all trials indicated significance. When doubling the treatment time to $96 \mathrm{~h}$ AVN-C again had the most profound effect, leaving just over $2 \%$ of observed cells in Q4. In addition, nearly $98 \%$ of cells stained positive for the presence of annexin V (Figs. 6b, c). Of the $98 \%$ annexin positive cells, a final $27 \%$ of cells were undergoing apoptosis while $71 \%$ were dead. Taken together, these time points indicate that AVN-C induces apoptosis beginning before $48 \mathrm{~h}$, and by 96 h viability has been reduced to 29 while $93 \%$ of cells still alive are on their way to an apoptotic death. At $400 \mu \mathrm{M}$ AVN-C and $96 \mathrm{~h}$ we found a greater number of dead cells than after treatment with STS, indicating that AVN-C is killing cells more effectively than our positive control. All AVN-C and STS treated quadrants were significantly different than vehicle. AVN-A and B also showed marked departure from the vehicle by $96 \mathrm{~h}$, in both a decrease in viable Q4 cells and an increase in apoptotic Q3 cells. Due to variance in the replicate experiments however, the $\mathrm{p}$ values indicated no significant difference in Q4 and Q3 as determined by a two tailed $\mathrm{T}$ test ( 0.054 and 0.08 respectively for AVN-A, and 0.092 in both quadrants for AVN-B).

To further confirm the apoptotic effects of AVN-C we analyzed the activity of caspases $3 / 7$. We treated cells with each AVN and STS, but analyzed the samples to detect the activity of caspase $3 / 7$, late stage apoptosis caspases (Fig. 7). As with the annexin V/PI co-staining, this assay allows the user to set up quadrants again detailing if cells are alive (Q4), dead (Q2), or undergoing apoptosis (Q3). For AVN-C, the results were nearly identical to those observed when staining with annexin V and PI at the same time point: $91 \%$ of cells showed caspase fluorescence and $8 \%$ of cells were found to be viable. Significance was observed in all three quadrants ( $\mathrm{p}$ values $4.99 \mathrm{E}-8$, 0.016, and 6.6E-8 for Q4, 3, and 2 respectively).

\section{Discussion}

AVN-C decreases the viability of MDA-MB-231 breast cancer cells in vitro through an apoptotic mechanism and not due to cell cycle arrest. As a result, we suggest AVN-C should be studied further as a chemotherapeutic. AVN-A and B also show some chemotherapeutic potential, but these effects are not nearly as pronounced 


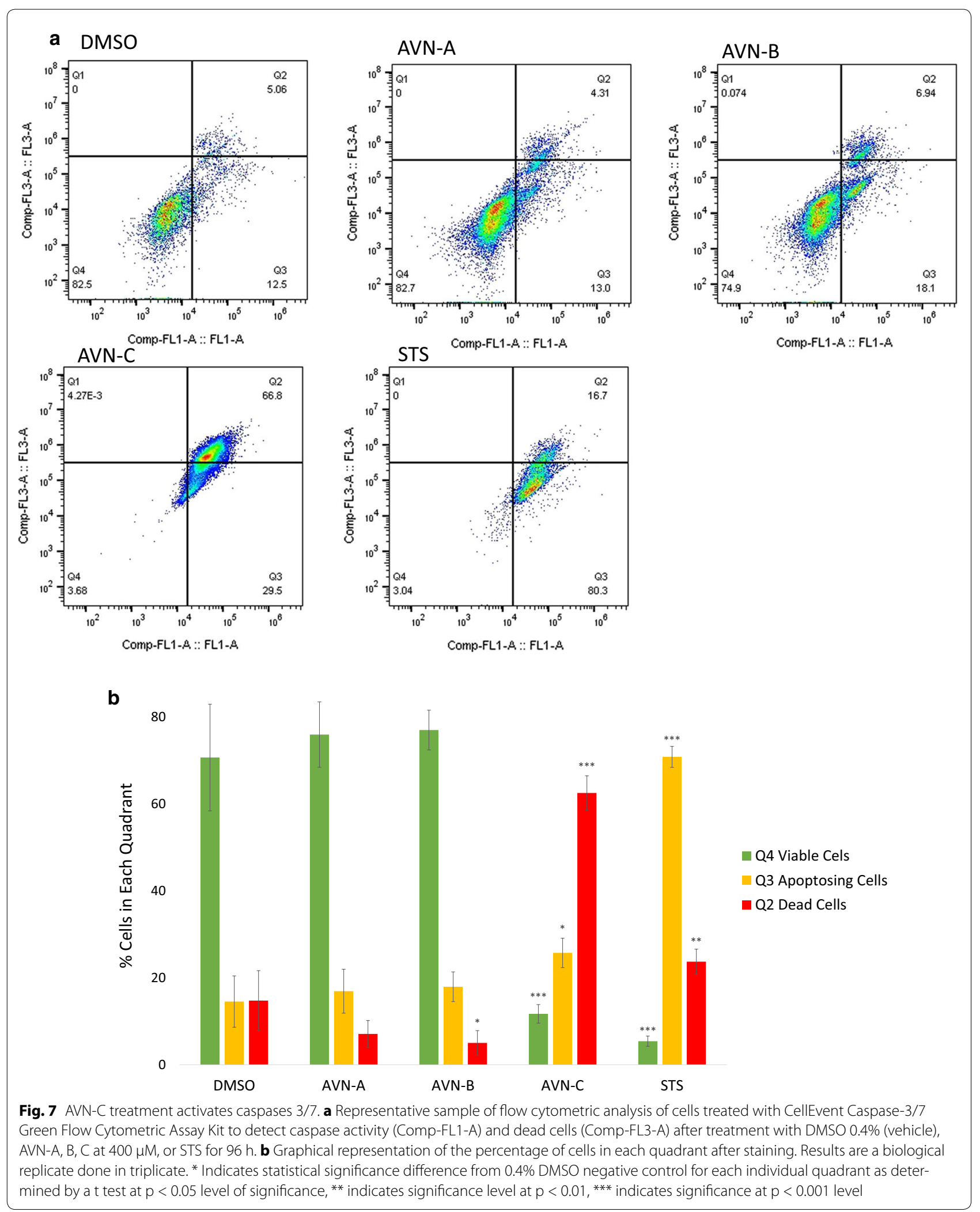


as those demonstrated by AVN-C, suggesting AVN-C is potentially the most effective of the three compounds in treating breast cancer.

Several plant-derived molecules have been shown to reduce cancer cell viability in a number of cancer cell lines [13]. These molecules are continually plagued by a high first pass metabolism and resulting low bioavailability, making them unfit chemotherapeutic agents [14]. AVN have high bioavailability [12] and activate apoptosis in breast cancer cells. As a result, they have the potential to act as effective chemotherapeutics. Unlike traditional chemotherapeutics AVN have not shown toxicity to normal cells; thus, AVN work as a chemotherapeutic that does not have the dose-limiting effects associated with current chemotherapy.

Chen et al. [12] demonstrated that the blood plasma levels increased by $3.3 \times$ for AVN-A, $7.3 \times$ for AVN-B, and $2.2 \times$ for $\mathrm{AVN}-\mathrm{C}$ in patients when the oral dose was doubled. This data indicates that higher initial doses of AVN may significantly increase the plasma concentration for AVN. Additionally, AVN have a 3-h half-life which is much longer than many plant based compounds. The bioavailability data presented by Chen et al. indicated that the high concentration of AVN seen to be effective in this study might be obtainable in humans. Further this study has only looked at the efficacy of AVN as a chemotherapeutic, and AVN might be an effective chemopreventative compound at lower doses.

In an effort to increase the treatment effectiveness, or decrease treatment consequences, of current chemotherapy AVN-C should be investigated further. To better understand the full chemotherapeutic potential of AVN, the pro-apoptotic activity of AVN should be tested in various other cancer cell lines, specifically colon cancer (CaCo-2 and HT29) and liver cancer (HepG2) cells where decreases in viability have already been demonstrated [8, 10]. These results should also be moved to in vivo studies with various doses and delivery options for AVN-C to find $\mathrm{IC}_{50}$ values fit for eventual human trials.

\section{Conclusions}

We conclude that AVN-C is a putative chemotherapeutic which causes apoptosis in MDA-MB-231 breast cancer cells. Without documented toxicity to normal cells AVN-C serves as a novel chemotherapeutic without dose-limiting side effects. It is of note that even though AVN-A and B did not activate apoptosis in a statistically significant manner, some caspase activity and positive annexin $\mathrm{V}$ staining was detected in our experiments.
Coupled with the significant decrease observed in viability in both a time and concentration dependent manner there is much that can still be learned from these molecules and their mechanisms of action in cancerous cells.

\begin{abstract}
Abbreviations
AVN: avenanthramide; Cmax: maximum plasma concentration; PI: propidium iodide; MTT: 3-(4,5-dimethylthiazol-2-yl)-2,5-diphenyltetrazolium bromide; NP40: Nonidet P-4; DMEM: Dulbecco's Modified Eagle Medium; FBS: fetal bovine serum; STS: staurosporine.
\end{abstract}

\section{Authors' contributions}

$\mathrm{JH}$ maintained cell culture, performed all experiments, and analyzed flow cytometry data in addition to writing the manuscript. JK designed the study, provided indispensable direction and insight, and participated in the editing of the manuscript. Both authors read and approved the final manuscript.

\section{Acknowledgements}

Thanks to the BYU RIC facility for use of their flow cytometer and data analysis software.

\section{Competing interests}

The authors declare that they have no competing interests.

Availability of data and materials

The data supporting the conclusions of this paper are included within the manuscript.

\section{Consent for publication}

Not applicable.

Ethics approval and consent to participate

Not applicable.

Funding

This work was supported by the BYU Life Science Start-up Grant.

\section{Publisher's Note}

Springer Nature remains neutral with regard to jurisdictional claims in published maps and institutional affiliations.

Received: 20 June 2017 Accepted: 13 October 2017

Published online: 18 October 2017

References

1. US breast cancer statistics. Breastcancer.org. 2017. http://www.breastcancer.org/symptoms/understand_bc/statistics. Accessed 14 May 2017.

2. Hanahan D, Weinberg RA. The hallmarks of cancer. Cell. 2000. doi:10.1016/ S0092-8674(00)81683-9.

3. Hanahan D, Weinberg RA. Hallmarks of cancer: the next generation. Cell. 2011. doi:10.1016/j.cell.2011.02.013.

4. Peterson DM. Oat antioxidants. J Cereal Sci. 2001. doi:10.1006/ jers.2000.0349.

5. Liu L, Zubik L, Collins FW, Marko M, Meydani M. The antiatherogenic potential of oat phenolic compounds. Atherosclerosis. 2004;175:1.

6. Nie L, Wise ML, Peterson D, Meydani M. Mechanism by which avenanthramide-c, a polyphenol of oats, blocks cell cycle progression in vascular smooth muscle cells. Free Radic Biol Med. 2006;41:702. 
7. Moglia A, Goitre L, Gianogli S, Baldini E, Trapani E, Genre A, Retta SF. Evaluation of the bioactive properties of avenanthramide analogs produced in recombinant yeast. BioFactors. 2015. doi:10.1002/biof.1197.

8. Guo W, Nie L, Wu D, Wise ML, Collins FW, Meydani SN, Meydani M. Avenanthramides inhibit proliferation of human colon cancer cell lines in vitro. Nutr Cancer. 2010. doi:10.1080/01635581.2010.492090.

9. Nie L, Wise ML, Peterson D, Meydani M. Avenanthramide, a polyphenol from oats, inhibits vascular smooth muscle cell proliferation and enhances nitric oxide production. Atherosclerosis. 2006. doi:10.1016/j. atherosclerosis.2005.07.027.

10. Scarpa ES, Antonini E, Palma F, Mari M, Ninfali P. Antiproliferative activity of vitexin-2-O-xyloside and avenanthramides on $\mathrm{CaCo}-2$ and $\mathrm{HepG} 2$ cancer cells occurs through apoptosis induction and reduction of prosurvival mechanisms. Eur J Nutr. 2017. doi:10.1007/s00394-017-1418-y.
11. Meydani M. Avenanthramides, unique polyphenols of oats with potential health effects. In: YiFang C, editor. Oats nutrition and technology. New York: Wiley; 2013. p. 255-64.

12. Chen YO, Milbury PE, Collins FW, Blumberg JB. Avenanthramides are bioavailable and have antioxidant activity in humans after acute consumption of an enriched mixture from oats. J Nutr. 2007;137:1375.

13. Zhou Y, Zheng J, Li Y, Xu DP, Li S, Chen YM, Li HB. Natural polyphenols for prevention and treatment of cancer. Nutrients. 2016;8:515. doi:10.3390/ nu8080515.

14. Biasutto L, Mattarei A, Sassi N, Azzolini M, Romio M, Paradisi C, Zoratti M. Improving the efficacy of plant polyphenols. Anticancer Agents Med Chem. 2014;14(10):1332-42. 